\title{
Síndrome de nistagmo vertical hacia abajo: Reporte de 2 casos y revisión de la literatura
}

\section{Downbeat nystagmus syndrome: A report of 2 cases and literature review}

\author{
Daniel Sotomayor J'1, Hayo Breinbauer K².
}

\begin{abstract}
RESUMEN
El síndrome de nistagmo vertical hacia abajo (NVA) es una forma común de nistagmo de fijación adquirido que se presenta con nistagmo persistente con fase rápida en dirección descendente, mareo, oscilopsia y alteraciones de la marcha. Se considera un trastorno vestíbulo-cerebelar debido a un defecto en las células de Purkinje en el flóculo del cerebelo. Las causas reportadas con mayor frecuencia son los trastornos degenerativos cerebelares e isquemia cerebelar, sin embargo, en un gran porcentaje de los pacientes la etiología permanece incierta (forma idiopática). EI NVA se puede dar en un contexto más amplio de neuropatía somatosensorial y ataxia cerebelar en el síndrome CANVAS. Las medidas terapéuticas incluyen evitar la posición supina y prona al descansar, rehabilitación vestibular y tratamiento farmacológico con aminopiridinas, entre otros. En este artículo presentamos dos casos de NVA así como la revisión de la literatura.
\end{abstract}

Palabras clave: Nistagmo vertical hacia abajo, ataxia cerebelar, rehabilitación vestibular, aminopiridinas.

\begin{abstract}
Downbeat nystagmus syndrome (DBN) is a frequent form of acquired fixation nystagmus, it presents with persisting nistagmus with fast phases directed downward, dizziness, oscillopsia and gait disturbances. It is considered a vestibulocerebellar disorder due to a bilateral defect of the Purkinje cells in the cerebellar flocculus. Most reported causes are degenerative disorders of the cerebellum and cerebellar ischemia, nevertheless the etiology remains unknown in a large percentage of patients (idiopathic form). DBN may present in a broader context of somatosensory neuropathy and cerebellar ataxia as in CANVAS syndrome. Therapeutic measures includes avoiding the supine and prone position when resting, vestibular rehabilitation, and pharmacologic treatment with aminopyridines, among others. In this article we present two cases of DBN and review of literature.
\end{abstract}

Key words: Downbeat nystagmus, cerebellar ataxia, vestibular rehabilitation, aminopyridine.

\footnotetext{
1 Interno de séptimo año de Medicina, Universidad del Desarrollo.

2 Médico Otorrinolaringólogo, ORL Clínica Alemana.

Recibido el 12 de julio de 2015. Aceptado el 21 de octubre de 2015.
} 


\section{INTRODUCCIÓN}

Uno de los desafíos de la otoneurología es el manejo de los trastornos de vértigo o mareo constantes, con sintomatología persistente durante todos 0 casi todos los días. En este grupo algunos cuadros centrales pueden tener una alta prevalencia y deben ser considerados dentro del diagnóstico diferencial. Presentamos dos casos de lo que corresponde a la causa más frecuente de nistagmo espontáneo y mareo persistente de origen central, ambos evaluados en el contexto del Centro del Vértigo y Trastornos del Equilibrio de Clínica Alemana.

\section{CASOS CLÍNICOS}

\section{CASO 1}

Paciente de 67 años, masculino, portador de hipertensión arterial manejado exitosamente con enalapril, previamente asintomático, consulta por cuadro de 6 meses de evolución y lentamente progresivo de mareo, inestabilidad y oscilopsia (pérdida del foco del campo visual con movimientos de cabeza) sobre todo en el eje vertical, e intolerancia al movimiento (sensación de que sus síntomas aumentan al girar la cabeza rápidamente en cualquier dirección). Los síntomas empeoran al estar en decúbito dorsal, por lo que prefiere dormir semisentado. En ningún momento hubo sensación de giro, y los síntomas se presentan por varias horas, fluctuantes, algo peor en la mañana, pero nunca llegando a la intensidad de ocasionar vómitos. Audición normal, clínicamente y a la audiometría, sin tinnitus.

Al examen físico destaca un nistagmo espontáneo inagotable vertical descendente, el cual aumenta de frecuencia e intensidad en posición supina (acostado, mirando hacia arriba) y aún más en cabeza colgando. En video oculografía su velocidad de fase lenta fue de $0,3 \%$ s sentado, $1,8 \%$ s acostado y $2,3 \%$ s con cabeza colgando. Dentro de su estudio presentó un video-impulso cefálico normal, pruebas posturales sin nistagmos posicionales propios de canalo o cupulolitiasis y resonancia nuclear magnética (RNM) de cerebro normal.

En base a la persistencia sin variación de nistagmo espontáneo vertical descendente en tres controles separados por 8 semanas, y la ausencia de otros hallazgos en su estudio, se determinó el diagnóstico de síndrome de nistagmo vertical hacia abajo (NVA) o downbeat nystagmus.

El paciente presentó inmediata mejoría sintomática con $5 \mathrm{mg}$ cada 12 hrs de fampridina (nombre comercial Fampyra ${ }^{\circledR}$, farmacológicamente también se le conoce como 4-aminopiridina), el cual ocupó durante un mes. Se discontinuó el uso del medicamento por su alto costo (sobre $\$ 250.000$.- para 40 días). Los síntomas regresaron una semana luego de suspender el medicamento. Tras 7 semanas de protocolo de rehabilitación vestibular de Clínica Alemana, con énfasis en ejercicios oculomotores y de integración oculomotora y de marcha, el paciente presentó mejora sintomática importante, refiriendo el problema como mínimo o inexistente en ya dos meses de seguimiento. En el último control, y a pesar de la mejoría sintomática, el nistagmo espontáneo descendente se mantenía presente sin grandes variaciones a las descritas en su evaluación inicial.

\section{CASO 2}

Paciente de 62 años, masculino. A los 32 años presentó un accidente automovilístico con traumatismo encefalocraneano, y cuadro de vértigo poscontusional. Se detectó una arreflexia vestibular derecha según prueba calórica. Tras el accidente realiza rehabilitación, con lo que la inestabilidad residual evoluciona favorablemente, logrando estar asintomático luego de algunos meses.

Se mantiene sin ninguna molestia por 3 décadas hasta hace 1 año, cuando comienza con inestabilidad, oscilopsia ocasional, pero sobre todo mareo persistente. No comenta mayor intolerancia al movimiento y no hay sensación de vértigo rotatorio en ningún momento.

Al examen físico destaca un nistagmo espontáneo vertical descendente, el cual aumenta de frecuencia e intensidad con cabeza colgando. En video oculografía su velocidad de fase lenta fue de $0,9 \%$ sentado y $2,4 \%$ acostado.

Dentro de su estudio, el video-impulso cefálico para canales laterales muestra sacadas correctivas overt bilaterales, con ganancias de reflejo vestíbuloocular de 0,44 a derecha y 0,61 a izquierda (hipofunción vestibular bilateral). RNM de cerebro normal. 
Dado los hallazgos descritos, se plantea también un síndrome NVA, considerando un CANVAS (síndrome de arreflexia vestibular bilateral, con neuropatía y ataxia cerebelar) incompleto (ver discusión).

Paciente declina intento de uso de fampridina por su alto costo. Se plantea uso de baclofeno $10 \mathrm{mg}$ al día, pero el paciente escoge intentar con rehabilitación vestibular. Tras 5 semanas de ejercicios semejantes a los del caso 1, paciente siente mejoría importante de su sintomatología, persistiendo mareo ocasional uno o dos días a la semana.

A los tres meses luego de completar su rehabilitación vestibular, en relación a un evento psicoemocional estresante, el paciente presenta una nueva exacerbación de sus síntomas, percibiendo en dos oportunidades crisis de segundos de duración de sensación de giro estando sentado con la vista al frente, sin gatillos posturales. Desde entonces percibe diplopía al llevar la mirada a la derecha extrema.

Luego de manejo con nuevas sesiones de rehabilitación vestibular, difenidol y benzodiacepinas (clonazepam) por dos semanas, el paciente vuelve a sentirse estable, con mínimo o ningún mareo. Hasta el último control tanto el nistagmo espontáneo vertical inagotable como la diplopía al llevar la mirada a derecha persistían sin cambios.

\section{DISCUSIÓN}

En ambos casos presentados, el elemento clínico común orientador es la presencia de un nistagmo vertical descendente persistente, inagotable. EI NVA o downbeat nystagmus es la forma adquirida (no congénita) más común de nistagmo de fijación ${ }^{1-3}$, presentándose en el $40 \%$ de los pacientes que presentan oscilopsia vertical. La edad promedio en 177 pacientes estudiados con NVA fue de 62 años ${ }^{4,5}$.

Clínicamente el NVA presenta una fase lenta donde los ojos se desvían hacia arriba seguido de una sacada correctiva en dirección inferior ${ }^{6}$. Generalmente se hace más intenso con la mirada lateral o hacia abajo, con la convergencia y al tenderse en posición prona (acostado, mirando hacia abajo) $)^{1,4}$, sin embargo, puede haber presentaciones atípicas donde la fase lenta se pronuncia incluso al mirar hacia arriba ${ }^{7}$. Este tipo de nistagmo no se suprime con la fijación de la mirada ${ }^{8}$. Los pacientes con NVA típicamente presentan mareo con la oscilopsia, especialmente con la mirada excéntrica, la mayoría refiere inestabilidad al estar de pie 0 al caminar, con aumento del balanceo postural anteroposterior $^{9-12}$. Como resultado de la oscilopsia vertical se produce alteración de la agudeza visual ${ }^{13,14}$. Se ha reportado que dentro del transcurso del día tanto la intensidad como los síntomas del NVA disminuyen en la tarde ${ }^{15}$, no obstante, la mayor parte de los pacientes refiere síntomas en forma permanente 0 casi permanente ${ }^{4}$. En relación a la marcha en casos de NVA se ha visto una reducción de la velocidad, con reducción en el largo de la zancada y aumento de la base de sustentación ${ }^{16}$. Es importante mencionar que el síndrome de NVA se acompaña muchas veces de otras alteraciones oculomotoras, especialmente paresias parciales de la mirada (como se ve en el fenómeno de diplopía al mirar a la derecha en el caso 2) y movimiento de búsqueda sacádico (en contraste con un seguimiento ocular de un objeto en movimiento fluido $)^{17}$.

El modelo fisiopatológico del NVA sugiere una disfunción de la región vestíbulo-cerebelar ${ }^{18}$. El flóculo del cerebelo inhibe el núcleo vestibular y mantiene los ojos estables durante la mirada hacia abajo. La desinhibición del flóculo lleva a una desviación lenta hacia arriba de los ojos, seguida de una sacada correctiva hacia abajo ${ }^{19}$. Se ha propuesto que esta desinhibición se debe a una alteración en la función de las células de Purkinje ubicadas en el flóculo cerebelar y de su asimetría fisiológica20,21, que al dañarse dejan de inhibir a las neuronas en el núcleo vestibular superior, llevando así a esta desviación espontánea hacia arriba de los ojos ${ }^{22}$. Los estudios de imagen cerebral y estudios de lesiones cerebelares dan evidencia más precisa de una hipofunción, hipometabolismo y reducción en el volumen de materia gris del flóculo y paraflóculo en estos pacientes ${ }^{23}$. Se plantea que hasta $50 \%$ de los pacientes presentarían evidencia en RNM de atrofia cerebelar, especialmente en línea media en las áreas del flóculo y paraflóculo, mientras que la otra mitad de los pacientes presentarían imágenes normales, como los dos casos aquí descritos.

La literatura muestra variadas causas de NVA, las reportadas con mayor frecuencia corresponden 
a patología cerebelar incluyendo trastornos degenerativos del cerebelo e isquemia cerebelar. Cabe destacar que en un alto porcentaje de los pacientes no se logra identificar una lesión anatómica, lo cual se conoce como NVA idiopático ${ }^{4,24}$. En un estudio se observó que la etiología se mantuvo incierta en $38 \%$ de los casos ${ }^{4}$. Hasta el presente se han reportado casos de NVA debido a efecto tóxico de fármacos como litio y carbamazepina, tumores cerebrales, trauma, encefalitis, esclerosis múltiple, lesiones paramedianas del bulbo raquídeo y malformación de Arnold-Chiari25-32.

El NVA se puede dar en el contexto de un síndrome más amplio cerebeloso-atáxico y somatosensorial neuropático en el CANVAS, en el cual se asocia disfunción vestibular bilateral y fenómenos neuropáticos en extremidades inferiores, pero también en términos de disfagia ${ }^{33}$. Dentro de este contexto se plantea que la degeneración a nivel cerebelar puede verse iniciado o incrementado por una paresia vestibular de otro origen, como en el caso del paciente número dos. En CANVAS es aún más frecuente encontrar otros fenómenos oculomotores de características cerebelares, como la paresia ocular parcial, que explica la diplopía al mirar en una dirección del segundo caso presentado. Sin embargo el paciente 2, no presenta fenómenos neuropáticos dentro de su historia, aunque se describe que los síntomas completos de CANVAS se pueden presentar hasta dentro de un plazo de 10 años $^{33}$.

Con referencia al tratamiento del NVA como de CANVAS, se han propuesto medidas generales, de rehabilitación vestibular kinésica y farmacológica.

Dentro de las generales, varios estudios analizaron que los síntomas son más intensos cuando el paciente está en decúbito prono que al estar parado, y que es más marcado luego de descansar en posición supina o prona por largos períodos durante el día. En estos casos, los síntomas visuales del nistagmo pueden minimizarse si el paciente descansa durante el día con la cabeza en posición vertical ${ }^{34,35}$.

Aunque no hay ensayos randomizados, la experiencia de distintos grupos con pacientes con NVA y CANVAS han comentado sistemáticamente que la rehabilitación vestibular y kinésica, aunque no disminuye las alteraciones oculomotoras características del cuadro, si son efectivas en disminuir la sintomatología al permitir una readecuación y compensación de la integración central de los componentes visuales, propioceptivos y vestibulares del equilibrio ${ }^{33,36}$.

Farmacológicamente se ha investigado la utilidad de distintos compuestos. Se generó un interés en el uso de agentes anticolinérgicos ${ }^{37}$, un estudio controlado doble ciego que utilizó trihexyphenidyl vio que se produjo sólo una modesta mejoría y efectos adversos que eran mal tolerados ${ }^{38}$. Se propuso utilizar fármacos gabaérgicos, una comparación doble ciego entre baclofeno y gabapentina mostró que ninguno de los dos produjo una mejoría consistente y que, en algunos pacientes, el nistagmo incluso empeoró ${ }^{39}$.

Sin embargo, otras experiencias apoyan el uso de baclofeno, el cual disminuiría la sintomatología de forma importante en dos tercios de los pacientes, mientras que en el resto no tendría ningún efecto, siendo considerado por mucho tiempo como una droga estándar para el intento terapéutico en estos pacientes ${ }^{33}$.

También se estudió el uso de clonazepam en 7 pacientes con NVA, se obtuvieron resultados favorables en los pacientes con NVA idiopático con reducción de la oscilopsia y mejora en la agudeza visual, en su contra está el hecho de que las benzodiacepinas con frecuencia causan sedación ${ }^{40}$.

Actualmente se ha adoptado una estrategia usando bloqueadores directos de canales iónicos para tratar el nistagmo $0^{41,42}$, donde las aminopiridinas, y en especial la 4-aminopiridina (4-AP 0 fampridina en nuestro país) y 3,4-diaminopiridina (3,4-DAP) han destacado por su efecto. Estas son bloqueadores no selectivos de canales de potasio voltaje dependientes ${ }^{43}$, se cree que aumentarían los niveles sinápticos del neurotransmisor inhibitorio ácido gamma-aminobutírico (GABA) en los sitios de proyección de las células de Purkinje ${ }^{19}$, incrementando su excitabilidad por prolongación y crecimiento del potencial de acción ${ }^{44}$, lo que aumentaría la influencia inhibitoria fisiológica alterada en las neuronas del núcleo vestibular superior ${ }^{45}$. Esto podría explicar la mejoría en la velocidad de la fase lenta del NVA. Estos efectos en el cerebelo fueron confirmados por un estudio con tomografía de emisión de positrones que mostró que la 4-AP incrementa la actividad metabólica bilateralmente en el flóculo ${ }^{46}$. 
Las aminopiridinas reducen la velocidad media de la fase lenta del NVA ${ }^{10,47-49}$. Un estudio clínico randomizado doble-ciego realizado en 27 pacientes con NVA que fueron tratados con 4-AP con dosis de 5 y $10 \mathrm{mg}$, mostró una mejoría en la velocidad de la fase lenta del nistagmo, que fue mayor utilizando la dosis baja. En el mismo trabajo se evidenció que la edad se correlacionaba en forma directa con un mayor efecto en la velocidad de la fase lenta y que el máximo beneficio los presentaron los pacientes con NVA idiopático. Además, se obtuvo mejoría en la posturografía, prueba de marcha y en la agudeza visual ${ }^{50}$. Comparando la eficacia de 4-AP con 3,4-DAP, la evidencia revela que al utilizar la misma dosis de ambas drogas la disminución de la velocidad media de la fase lenta medida con oculografía 3D es significativamente mayor con la primera ${ }^{51}$. Una alternativa pudiese ser el uso de dalfampridine, la versión de liberación sostenida de la 4-AP, que ha mostrado ser efectivo, con una mayor tasa de pacientes que responden al tratamiento que 4-AP y con pocos efectos adversos ${ }^{52}$. Por otro lado, se ha visto que en pacientes con ataxia episódica tipo 2, las aminopiridinas también reducen la ataxia cerebelar intercrisis y la frecuencia de los ataques ${ }^{53,54}$. La 4-AP, más estudiada, se absorbe en forma rápida, con un nivel sérico máximo entre 20 a 60 minutos ${ }^{55}$, está contraindicada ante síndrome de QT largo por 10 que debiera pedirse un electrocardiograma antes y después de la primera administración. Debido a que este medicamento sólo tiene un efecto sintomático, se requiere de su utilización en forma continua ${ }^{8}$.

Por otra parte, se han reportado resultados satisfactorios de pacientes con NVA manejados en forma quirúrgica (ver referencias para detalles), sin embargo, no debería considerarse como primera opción $n^{56,57}$.

Con respecto al pronóstico, se sabe poco acerca de la historia natural en casos de NVA idiopático. Un estudio que siguió a pacientes con NVA idiopático hasta por 6 años mostró que no hubo cambios significativos en la velocidad de la fase lenta durante el lapso de tiempo investigado ${ }^{58}$.

\section{CONCLUSIÓN}

Los cuadros centrales de vértigo y mareo, especialmente aquellos que presentan sintomatología persistente son un desafío importante para el médico que trata a estos pacientes. Conocer la existencia de los cuadros de NVA o downbeat nystagmus syndrome y el CANVAS, permiten al clínico tener una mirada abierta y poder identificar estos cuadros, pudiendo acoger al paciente en la realidad, de que aunque presente un cuadro de características centrales, éste no representa necesariamente una patología maligna, desmielinizante o tumoral sino una patología neurodegenerativa idiopática.

El cuadro clínico, con sus elementos de nistagmo descendente persistente, la ausencia de otra sintomatología neurológica aparte de la descrita en la revisión, imágenes normales o que demuestren atrofia de las estructuras de línea media del cerebelo, parecen ser suficientes para esbozar este diagnóstico.

Dentro del tratamiento, destaca el uso de fampridina y en segundo término baclofeno dentro de las alternativas farmacológicas, donde sobre todo el primero actuaría presuntamente corrigiendo la alteración en su origen cerebelar. Lamentablemente, sobre todo la fampridina sigue siendo un medicamento de alto costo. Por otro lado, y como fue posible evidenciar en nuestros casos, la rehabilitación vestibular aunque no resuelve los fenómenos oculomotores, tiene un rol más que importante en la mejoría sintomática de estos pacientes.

\section{BIBLIOGRAFÍA}

1. Leigh RJ, Zee DS. The Neurology of Eye Movements. 4th ed. New York: Oxford University Press 2006; 405-63.

2. Thurtell MJ, Leigh RJ. Nystagmus and saccadic intrusions. Handbook of Clinical Neurology: Neuro-Ophthalmology. Amsterdam Elsevier 2011; 333-78.

3. Sarvananthan N, Surendran M, Roberts EO, et al. The prevalence of nystagmus: the Leicestershire nystagmus survey. Invest Ophthalmol Vis Sci 2009; 50(11): 5201-6.

4. Wagner JN, Glaser M, Brandt T, Strupp M. Downbeat nystagmus: aetiology and comorbidity in 117 patients. J Neurol Neurosurg Psychiatry 2008; 79(6): 672-7.

5. BALOH RW, SPOONER JW. Downbeat nystagmus: a type of central vestibular nystagmus. Neurology 1981; 31(3): 304-10. 
6. LEIGH RJ. Potassium channels, the cerebellum, and treatment for downbeat nystagmus. Neurology 2003; 61(2): 158-9.

7. Schmidt D. Downbeat nystagmus an upgaze. Neuro-Ophthalmology 1991; 11: 223-7.

8. Strupp M, Hüfner K, Sandmann R, et al. Central oculomotor disturbances and nystagmus: a window into the brainstem and cerebellum. Dtsch Arztebl Int 2011; 108(12): 197-204.

9. Büchele W, Brandt T, Degner D. Ataxia and oscillopsia in downbeat-nystagmus vertigo syndrome. Adv Otorhinolaryngol 1983; 30: 2917.

10. Helmchen C, Sprenger A, Rambold H, Sander T, Kömpf D, Straumann D. Effect of 3,4-diaminopyridine on the gravity dependence of ocular drift in downbeat nystagmus. Neurology 2004; 63(4): 752-3.

11. Pierrot-Deseilligny C, Milea D. Vertical nystagmus: clinical facts and hypotheses. Brain 2005; 128(Pt 6): 1237-46.

12. Sprenger A, Zils E, Rambold H, Sander T, HeLmchen C. Effect of 3,4-diaminopyridine on the postural control in patients with downbeat nystagmus. Ann N Y Acad Sci 2005; 1039: 395-403.

13. Bronstein AM. Vision and vertigo: some visual aspects of vestibular disorders. J Neurol 2004; 251(4): 381-7.

14. Kalla R, Glasauer S, Büttner U, Brandt T, Strupp M. 4-aminopyridine restores vertical and horizontal neural integrator function in downbeat nystagmus. Brain 2007; 130(Pt 9): 2441-51.

15. Spiegel R, Rettinger N, Kalla R, et al. The intensity of downbeat nystagmus during daytime. Ann N Y Acad Sci 2009; 1164: 293-9.

16. Schniepp R, Wuehr M, Huth S, et al. The gait disorder in downbeat nystagmus syndrome. PLoS One 2014; 9(8): e105463.

17. Glasauer S, Hoshi M, Kempermann U, Eggert T, BÜTTNER U. Three-dimensional eye position and slow phase velocity in humans with downbeat nystagmus. J Neurophysiol 2003; 89(1): 33854.

18. Zee DS, Yamazaki A, Butler PH, Gücer G. Effects of ablation of flocculus and paraflocculus of eye movements in primate. J Neurophysiol 1981; 46(4): 878-99.

19. Shalkh AG. Does 4-aminopyridine "beat" downbeat nystagmus? J Neurol Neurosurg Psychiatry 2013; 84(12): 1298-9.

20. Kalla R, Deutschlander A, Hufner K, et al. Detection of floccular hypometabolism in downbeat nystagmus by fMRI. Neurology 2006; 66(2): 281-3.

21. Glasauer S, Strupp M, Kalla R, Büttner U, Brandt T. Effect of 4-aminopyridine on upbeat and downbeat nystagmus elucidates the mechanism of downbeat nystagmus. Ann N Y Acad Sci2005; 1039: 528-31.

22. Marti S, Straumann D, Büttner U, Glasauer S. A model-based theory on the origin of downbeat nystagmus. Exp Brain Res 2008; 188(4): 61331.

23. Hüfner K, Stephan T, Kalla R, et al. Structural and functional MRIs disclose cerebellar pathologies in idiopathic downbeat nystagmus. Neurology 2007; 69(11): 1128-35.

24. Halmagy GM, Rudge P, Gresty MA, Sanders MD. Downbeating nystagmus. A review of 62 cases. Arch Neurol 1983; 40(13): 777-84.

25. Schмidt D. Downbeat-Nystagmus. A clinical review. Neuro-Ophthalmology 1991; 11: 24762.

26. Monden MA, Nederkoorn PJ, Tissma M. Downbeat nystagmus - a rare side-effect of lithium carbonate. Tijdschr Psychiatr 2015; 57(1): 4953.

27. Chrousos GA, Cowdry R, Schuelein M, Abdul-Rahim AS, Matsuo V, Currie JN. Two cases of downbeat nystagmus and oscillopsia associated with carbamazepine. Am J Ophthalmol 1987; 103(2): 221-4.

28. Lea J, Lechner C, Halmagyi GM, Welgampola MS. Not so benign positional vertigo: paroxismal downbeat nystagmus from a superior cerebellar peduncle neoplasm. Otol Neurotol 2014; 35(6): e204-5.

29. Hirst LW, ClaRk AW, WolisnKy JS, et Al. Downbeat nystagmus. A case report of herpetic brain stem encephalitis. J Clin Neuroophthalmo/ 1983; 3(4): 245-9.

30. Masucci EF, KuRTZke JF. Downbeat nystagmus secundary to multiple sclerosis. Ann Ophthalmol 1988; 20(9): 347-8.

31. Nakamagoe K, Shimizu K, Koganezawa T. Downbeat nystagmus due to a paramedian medullary lesión. J Clin Neurosci 2012; 19(11): 1597-9. 
32. Wagner J, Lehnen N, Glasauer S, et al. Downbeat nystagmus caused by a paramedian pontomedullary lesion. J Neurol 2009; 256(9): 15724.

33. Szmulewicz DJ, Mclean CA, MacDougall HG, Roberts L, Storey E, Halmagy GM. CANVAS an update: clinical presentation, investigation and management. J Vestib Res 2014; 24(5-6): 46574.

34. ThuRTELL MJ, LEIGH RJ. Treatment of nystagmus. Curr Treat Options Neurol 2012; 14(1): 6072.

35. Spiegel R, Kalla R, Rettinger N, et al. Head position during resting modifies spontaneous daytime decrease of downbeat nystagmus. Neurology 2010; 75(21): 1928-32.

36. Porciuncula F, Johnson CC, Glickman LB. The effect of vestibular rehabilitation on adults with bilateral vestibular hypofunction: a systematic review. $J$ Vestib Res 2012; 22(5-6): 283-98.

37. Barton JJ, Huaman AG, Sharpe JA. Muscarinic antagonists in the treatment of acquired pendular and downbeat nystagmus: a double-blind, randomized trial of three intravenous drugs. Ann Neurol 1994; 35(3): 319-25.

38. Leigh RJ, Burnstine TH, Ruff RL, Kasmer RJ. The effect of anticholinergic agents upon acquired nystagmus: a double-blind study of trihexyphenidyl and tridihexethyl chloride. Neurology 1991; 41(11): 1737-41.

39. Averbuch-Heller L, Tusa RJ, Rottach KG, et al. A double-blind controlled study of gabapentin and baclofen as treatment for acquired nystagmus. Ann Neurol 1997; 41(6): 818-25.

40. Young YH, Huang TW. Role of clonazepam in the treatment of idiopathic downbeat nystagmus. Laryngoscope 2001; 111(8): 1490-3.

41. Sedehizadeh S, Keogh M, Maddison P. The use of aminopyridines in neurological disorders. Clin Neuropharmacol 2012; 35(4): 191-200.

42. Strupp M, Kalla R, Glasauer S, et al. Aminopyridines for the treatment of cerebellar and ocular motor disorders. Prog Brain Res 2008; 171: 535-41.

43. Strupp M, Feil K, Bardins S, Waidelich R. 4-aminopyridine improves lower urinary tract symptoms in a patient with benign prostatic hyperplasia and downbeat nystagmus syndrome. Int Neurourol J 2014; 18(4): 221-5.
44. Alviña K, Khodakhah K. The therapeutic mode of action of 4-aminopyridine in cerebellar ataxia. $J$ Neurosci 2010; 30(21): 7258-68.

45. Etzion Y, Grossman Y. Highly 4-aminopyridine sensitive delayed rectifier current modulates the excitability of guinea pig cerebellar Purkinje cells. Exp Brain Res 2001; 139(4): 419-25.

46. Brense S, Best C, Buchholz HG, et al. 18F-fluorodeoxyglucose hypometabolism in cerebellar tonsil and flocculus in downbeat nystagmus. Neuroreport 2006; 17(6): 599603.

47. Strupp M, Schüler 0, Krafczyk S, et al. Treatment of downbeat nystagmus with 3,4-diaminopyridine: a placebo controlled study. Neurology 2003; 61(2): 165-70.

48. Kalla R, Glasauer S, Schautzer F, et al. 4-aminopyridine improves downbeat nystagmus, smooth pursuit, and VOR gain. Neurology 2004; 62(7): 1228-9.

49. Sprenger A, Rambold H, Sander T, et al. Treatment of the gravity dependence of downbeat nystagmus with 3,4-diaminopyridine. Neurology 2006; 67(5): 905-7.

50. Claasen J, Spiegel R, Kalla R, et al. A randomised double-blind, cross-over trial of 4-aminopyridine for downbeat nystagmus - effects on slowphase eye velocity, postural stability, locomotion and symptoms. J Neurol Neurosurg Psychiatry 2013; 84(12): 1392-9.

51. Kalla R, Spiegel R, Claassen J, et al. Comparison of $10-\mathrm{mg}$ doses of 4-aminopyridine and 3,4-diaminopyridine for the treatment of downbeat nystagmus. J Neuroophthalmo/2011; 31(4): 320-5.

52. Claassen J, Feil K, Bardins S, et al. Dalfampridine in patients with downbeat nystagmus - an observational study. J Neurol 2013; 260(8): 1992-6.

53. Strupp M, Kalla R, Dichgans M, Freilinger T, Glasauer S, Brandt T. Treatment of episodic ataxia type 2 with the potassium channel blocker 4-aminopyridine. Neurology 2004; 62(9): 1623-5.

54. Löhle M, SchrempF W, Wolz M, Reichmann H, Storch A. Potassium channel blocker 4-aminopyridine is effective in interictal cerebellar symptoms in episodic ataxia type 2 - a video case report. Mov Disord 2008; 23(9): 1314-6. 
55. Judge SI, Bever CT Jr. Potassium channel blockers in multiple sclerosis: neuronal Kv channels and effects of symptomatic treatment. Pharmacol Ther 2006; 111(1): 224-59.

56. Jain S, Proudlock F, Constantinescu CS, Gottlob I. Combined pharmacologic and surgical approach to acquired nystagmus due to multiple sclerosis. Am J Ophthalmol 2002; 134(5): 780-2.

57. Wang ZI, Dell'Osso LF, Tomsak RL, Jacobs
JB. Combining recessions (nystagmus and strabismus) with tenotomy improved visual function and decreased oscillopsia and diplopia in acquired downbeat nystagmus and in horizontal infantile nystagmus síndrome. $J$ AAPOS 2007; 11(2): 135-41.

58. Wagner J, Lehnen N, Glasauer S, Strupp M, Brandt T. Prognosis of idiopathic downbeat nystagmus. Ann N Y Acad Sci 2009; 1164: 479-81. 